TRANSACTIONS OF THE

AMERICAN MATHEMATICAL SOCIETY

Volume 352 , Number 6 , Pages 2581-2600

S 0002-9947(00)02386-2

Article electronically published on February 14, 2000

\title{
PARTITIONS INTO PRIMES
}

\author{
YIFAN YANG
}

\begin{abstract}
We investigate the asymptotic behavior of the partition function $p_{\Lambda}(n)$ defined by $\sum_{n=0}^{\infty} p_{\Lambda}(n) x^{n}=\prod_{m=1}^{\infty}\left(1-x^{m}\right)^{-\Lambda(m)}$, where $\Lambda(n)$ denotes the von Mangoldt function. Improving a result of Richmond, we show that $\log p_{\Lambda}(n)=2 \sqrt{\zeta(2) n}+O\left(\sqrt{n} \exp \left\{-c(\log n)\left(\log _{2} n\right)^{-2 / 3}\left(\log _{3} n\right)^{-1 / 3}\right\}\right)$, where $c$ is a positive constant and $\log _{k}$ denotes the $k$ times iterated logarithm. We also show that the error term can be improved to $O\left(n^{1 / 4}\right)$ if and only if the Riemann Hypothesis holds.
\end{abstract}

\section{INTRODUCTION}

The asymptotic behavior of partition functions has been extensively studied in the literature. The most famous result is the asymptotic formula

$$
p(n) \sim \frac{1}{(4 \sqrt{3}) n} e^{\pi \sqrt{2 n / 3}} \quad(n \rightarrow \infty)
$$

for the ordinary partition function $p(n)$, proved in 1918 by Hardy and Ramanujan [3]. The asymptotic behavior of more general partition functions has been studied by many authors, including Ingham [4], Kohlbecker [6], Meinardus [7], [8], Roth and Szekeres 12, Schwarz 13, 14, and Richmond 10, 11.

Of particular interest are functions related to partitions into primes. As an application of an asymptotic formula for general partition functions, Roth and Szekeres [12] showed that the number $q_{\mathcal{P}}(n)$ of partitions of $n$ into distinct primes satisfies

$$
\log q_{\mathcal{P}}(n)=\pi \sqrt{\frac{2}{3}}\left(\frac{n}{\log n}\right)^{1 / 2}\left\{1+O\left(\frac{\log \log n}{\log n}\right)\right\} .
$$

A similar, but in some ways more natural, partition function is the function $p_{\Lambda}(n)$ defined by

$$
\sum_{n=0}^{\infty} p_{\Lambda}(n) x^{n}=\prod_{m=1}^{\infty}\left(1-x^{m}\right)^{-\Lambda(m)}
$$

where

$$
\Lambda(m)= \begin{cases}\log p, & m=p^{r}, \\ 0, & \text { else, }\end{cases}
$$

is the von Mangoldt function. The function $p_{\Lambda}(n)$ represents a weighted count of the number of partitions of $n$ into prime powers. This weighted partition function was first introduced and studied in 1950 by Brigham [1], who proved a conditional

Received by the editors March 3, 1998.

2000 Mathematics Subject Classification. Primary 11P82; Secondary 11M26, 11 N05. 
result under the assumption of the Riemann Hypothesis. More recently, Richmond [11], using an asymptotic formula for general partition functions (see [10]) and Vinogradov's zero-free region for the Riemann zeta function, obtained the following unconditional result.

Theorem A (Richmond). There exists a positive constant $c$ such that for all sufficiently large $n$

$$
\log p_{\Lambda}(n)=2 \sqrt{\zeta(2)} n^{1 / 2}\left(1+O\left\{\exp \left(-c(\log n)^{4 / 7}(\log \log n)^{-3 / 7}\right)\right\}\right) .
$$

Richmond also proved a conditional result.

Theorem B (Richmond). Let $\theta$ be the least upper bound for the real parts of the non-trivial zeros of the Riemann zeta function. Then

$$
\log p_{\Lambda}(n)=2 \sqrt{\zeta(2)} n^{1 / 2}+O\left(n^{\theta / 2}\right) .
$$

In particular, if the Riemann Hypothesis is true, then

$$
\log p_{\Lambda}(n)=2 \sqrt{\zeta(2)} n^{1 / 2}+O\left(n^{1 / 4}\right) .
$$

In our first result we show that the error term in Richmond's unconditional result (1.1) can be substantially improved.

Theorem 1. There exists a positive constant $c$ such that for all sufficiently large $n$

$$
\log p_{\Lambda}(n)=2 \sqrt{\zeta(2)} n^{1 / 2}\left\{1+O\left(\exp \left\{-\frac{c \log n}{\left(\log _{2} n\right)^{2 / 3}\left(\log _{3} n\right)^{1 / 3}}\right\}\right)\right\},
$$

where $\log _{k}$ denotes the $k$ times iterated logarithm.

Note that the exponential factor in (1.4) is much smaller than the corresponding factor in the error term of the prime number theorem obtained by using the Vinogradov zero-free region, namely $\exp \left(-(\log n)^{3 / 5-\epsilon}\right)$. The reason for this unexpectedly small error term lies in the fact that $\log p_{\Lambda}(n)$ behaves in many respects more like the power series $f(x)=\sum_{n=1}^{\infty} \Lambda(n) x^{n}$ than the partial sum $\Psi(u)=\sum_{n \leq u} \Lambda(n)$; indeed, it would not be hard to show that, as $x \rightarrow 1-, f(x)$ differs from its approximation $1 /(1-x)$ by a similarly small error term.

While the estimate of Theorem A can be substantially improved, our next result shows that Theorem B is best possible.

Theorem 2. We have

$$
\log p_{\Lambda}(n)-2 \sqrt{\zeta(2)} n^{1 / 2}=\Omega_{ \pm}\left(n^{1 / 4}\right) .
$$

Our final result gives a converse to Theorem B.

Theorem 3. Let $\theta$ be the least upper bound for the real parts of the zeros of the Riemann zeta function, and let $\theta^{\prime}$ be the greatest lower bound for all $\alpha$ for which

$$
\log p_{\Lambda}(n)=2 \sqrt{\zeta(2)} n^{1 / 2}+O\left(n^{\alpha / 2}\right)
$$

as $n \rightarrow \infty$. Then $\theta \leq \theta^{\prime}$.

Combining this result with Theorem B yields the following corollary. 
Corollary. Let $\theta$ and $\theta^{\prime}$ be defined as in Theorem 3. Then $\theta=\theta^{\prime}$. In particular, the Riemann Hypothesis is true if and only if

$$
\log p_{\Lambda}(n)=2 \sqrt{\zeta(2)} n^{1 / 2}+O_{\epsilon}\left(n^{1 / 4+\epsilon}\right)
$$

for all $\epsilon>0$.

Let

$$
P_{\Lambda}(u)=\sum_{n \leq u} p_{\Lambda}(n)
$$

be the summatory function of $p_{\Lambda}(n)$. In Section 4 we show that Theorems 1-3 are true if and only if the corresponding statements with $n$ replaced by $u$ and $p_{\Lambda}(n)$ replaced by $P_{\Lambda}(u)$ are true. Hence it suffices to prove Theorems 1-3 with $P_{\Lambda}(u)$ instead of $p_{\Lambda}(n)$.

Our main tools for proving these results are Abelian and Tauberian theorems that relate estimates for $P_{\Lambda}(u)$ to estimates for the Laplace-Stieltjes transform of $P_{\Lambda}(u)$, defined by

$$
F_{\Lambda}(x)=\int_{0}^{\infty} e^{-x u} d P_{\Lambda}(u) .
$$

We will state and prove these results in Section 2. In Section 3 we establish some lemmas relating the behavior of general weighted partition functions $p_{w}(n)$ defined by

$$
\sum_{n=0}^{\infty} p_{w}(n) x^{n}=\prod_{m=1}^{\infty}\left(1-x^{m}\right)^{-w(m)}
$$

to analytic properties of the Dirichlet series

$$
f_{w}(s)=\sum_{n=1}^{\infty} \frac{w(n)}{n^{s}},
$$

where $w(m)$ is a non-negative function defined on the set of positive integers. Our methods here are, to some extent, similar to those in Meinardus [7]. However, Meinardus made much stronger assumptions on the analytic properties of $f_{w}(s)$. These assumptions are not satisfied in the case $w(n)=\Lambda(n)$, so that Meinardus' results are not applicable to $p_{\Lambda}(n)$. In Section 4 we complete the proof of Theorems 1-3. In Section 5 we use one of our Tauberian results to give a new proof of Theorem B that is simpler and more elementary than the original proof of Richmond.

\section{Some Abelian and Tauberian Results}

Our first result is an elementary Abelian result which generalizes and extends a result of Freiman [2, p. 276].

Proposition 1. Suppose that $P(u)$ is a non-negative and non-decreasing function satisfying $P(t)=O_{\epsilon}\left(e^{\epsilon t}\right)$ for every $\epsilon>0$. For $x>0$ let

$$
F(x)=\int_{0}^{\infty} e^{-x u} d P(u)
$$

be the Laplace-Stieltjes transform of $P(u)$. Suppose that for some constants $A>0$ and $0<a<1$ the inequality

$$
\log P(u) \geq A u^{a}-r(u)
$$


holds for all sufficiently large $u$, where $r(u)$ is a positive differentiable function satisfying

$$
r(u) u^{-a} \rightarrow 0 \text { monotonically as } u \rightarrow \infty
$$

and

$$
r(u) /(\log u) \rightarrow \infty \text { monotonically as } u \rightarrow \infty
$$

Then

$$
\log F(x) \geq B x^{-b}-r\left(B^{\prime} x^{-b-1}\right)
$$

for all sufficiently small $x$, where

$$
B=(A a)^{1 /(1-a)}(1-a) / a, \quad b=a /(1-a), \quad B^{\prime}=B b .
$$

Similarly, if

$$
\log P(u) \leq A u^{a}+r(u)
$$

for all sufficiently large $u$, then with the same notation

$$
\log F(x) \leq B x^{-b}+2 r\left(B^{\prime} x^{-b-1}\right)
$$

for all sufficiently small $x$.

As a simple consequence of Proposition 1, we have the following corollary.

Corollary. Let $P(u), F(x), A, a, B, b$ and $B^{\prime}$ be given as in Proposition 1. Suppose that $r(u)$ satisfies conditions $(\mathrm{R} 1)$ and $(\mathrm{R} 2)$. Then

$$
\log F(x)-B x^{-b}=\Omega_{+}\left(r\left(B^{\prime} x^{-b-1}\right)\right)
$$

as $x \rightarrow 0$ implies that

$$
\log P(u)-A u^{a}=\Omega_{+}(r(u))
$$

as $u \rightarrow \infty$. The same statement holds if $\Omega_{+}$is replaced by $\Omega_{-}$.

Proof of Proposition 1. Suppose first that (2.1a) holds for sufficiently large $u$. By the monotonicity of $P(t)$ and the assumption that $P(t)$ is non-negative, we have for all $x>0$ and all $u>0$,

$$
\begin{aligned}
F(x) & =\int_{0}^{\infty} e^{-x t} d P(t)=x \int_{0}^{\infty} e^{-x t} P(t) d t \\
& \geq x \int_{u}^{\infty} e^{-x t} P(t) d t \geq x \int_{u}^{\infty} e^{-x t} P(u) d t=e^{-x u} P(u) .
\end{aligned}
$$

(Note that the convergence of the integral defining $F(x)$ is ensured by the assumption that $P(t)=O_{\epsilon}\left(e^{\epsilon t}\right)$ for every $\epsilon>0$.)

By (2.1a) it follows that

$$
F(x) \geq \exp \left\{-x u+A u^{a}-r(u)\right\}
$$

for all sufficiently large $u$ and all $x>0$. We choose $u=u_{x}$ to maximize $-x u+A u^{a}$, i.e., we let $u_{x}$ be defined by

$$
x=\operatorname{Aau}_{x}^{-(1-a)} .
$$

If $x$ is sufficiently small, then $u_{x}$ will be large enough for (2.5) to hold. We note that

$$
x u_{x}=x\left(A a x^{-1}\right)^{1 /(1-a)}=B^{\prime} x^{-a /(1-a)}=B^{\prime} x^{-b}
$$


and

$$
A u_{x}^{a}=A\left(A a x^{-1}\right)^{a /(1-a)}=(A a)^{1 /(1-a)} a^{-1} x^{-b}=B^{\prime} a^{-1} x^{-b} .
$$

Substituting these expressions into the right-hand side of (2.5), we obtain

$$
\log F(x) \geq B^{\prime}\left(-1+a^{-1}\right) x^{-b}-r\left(B^{\prime} x^{-b-1}\right)=B x^{-b}-r\left(B^{\prime} x^{-b-1}\right),
$$

which proves (2.2a). It remains to prove that (2.1b) implies $(2.2 \mathrm{~b})$.

Let $u_{0}$ be a positive constant such that (2.1b) and conditions (R1)-(R2) are satisfied for $u \geq u_{0}$. Given a positive number $x$, we let $u_{x}$ be defined by (2.6) and assume that $x$ is small enough so that $u_{x} \geq 2 u_{0}$. We write

$$
\begin{aligned}
F(x) & =x\left\{\int_{0}^{u_{0}}+\int_{u_{0}}^{(1-\mu) u_{x}}+\int_{(1-\mu) u_{x}}^{(1+\mu) u_{x}}+\int_{(1+\mu) u_{x}}^{\infty}\right\} e^{-x t} P(t) d t \\
& =x\left\{I_{1}+I_{2}+I_{3}+I_{4}\right\},
\end{aligned}
$$

where $\mu$ is a parameter to be chosen so that $x I_{3}$ has the same order of magnitude as $F(x)$. It turns out that the optimal choice for $\mu$ is

$$
\mu=\mu\left(u_{x}\right)=K \sqrt{u_{x}^{-a} r\left(u_{x}\right)}
$$

where $K$ is a positive constant to be chosen later in terms of $A$ and $a$. We note that condition (R1) implies that $\mu\left(u_{x}\right) \rightarrow 0$ as $u_{x} \rightarrow \infty$. In particular, we have $0<\mu<1 / 2$ if $x$ is sufficiently small in terms of $K$, which we will henceforth assume.

The integral $I_{1}$ is bounded by $\int_{0}^{u_{0}} P(t) d t$ and thus of order $O(1)$. To estimate $I_{4}$, we define $\phi(t)$ for $t \geq u_{0}$ by

$$
\phi(t)=-x t+A t^{a}+r(t) .
$$

Since, by condition (R1), $r(t) t^{-a}$ is monotonically decreasing to 0 , we have $r^{\prime}(t) t^{-a}$ $a r(t) t^{-a-1} \leq 0$ and $r(t) t^{-1} \leq r\left(u_{x}\right) u_{x}^{-a} u_{x}^{-(1-a)}=K^{-2} \mu^{2} u_{x}^{-(1-a)}$ for all $t \geq u_{x}$. Thus, for all $t \geq(1+\mu) u_{x}$ we obtain

$$
\begin{aligned}
\phi^{\prime}(t) & =-x+A a t^{-(1-a)}+r^{\prime}(t) \leq-x+A a t^{-(1-a)}+a r(t) t^{-1} \\
& \leq-x+A a(1+\mu)^{-(1-a)} u_{x}^{-(1-a)}+K^{-2} a \mu^{2} u_{x}^{-(1-a)} .
\end{aligned}
$$

By (2.6), the last expression is equal to $\left(-1+(1+\mu)^{-(1-a)}+O\left(\mu^{2}\right)\right) x$. Since $(1+\mu)^{-(1-a)}=1-(1-a) \mu+O\left(\mu^{2}\right)$, we have $-1+(1+\mu)^{-(1-a)}+O\left(\mu^{2}\right) \leq-(1-a) \mu / 2$ for all sufficiently small $x$. Hence for all sufficiently small $x$ and $t \geq(1+\mu) u_{x}$ we obtain

It follows that

$$
\phi^{\prime}(t) \leq-\frac{1-a}{2} x \mu
$$

$$
\begin{aligned}
x I_{4} & \leq x \int_{(1+\mu) u_{x}}^{\infty} e^{\phi(t)} d t \leq-\frac{2}{(1-a) \mu} \int_{(1+\mu) u_{x}}^{\infty} e^{\phi(t)} \phi^{\prime}(t) d t=\frac{2}{(1-a) \mu} e^{\phi\left((1+\mu) u_{x}\right)} \\
& =\frac{2}{(1-a) \mu} \exp \left\{-x(1+\mu) u_{x}+A(1+\mu)^{a} u_{x}^{a}+r\left((1+\mu) u_{x}\right)\right\} .
\end{aligned}
$$

We substitute (2.7) and (2.8) into the last expression and note that condition (R1) implies that $r\left((1+\mu) u_{x}\right)\left((1+\mu) u_{x}\right)^{-a} \leq r\left(u_{x}\right) u_{x}^{-a}$, i.e.,

$$
r\left((1+\mu) u_{x}\right) \leq(1+\mu)^{a} r\left(u_{x}\right)
$$


for all sufficiently small $x$. This yields

$$
\begin{aligned}
x I_{4} \leq & \frac{2}{(1-a) \mu} \exp \left\{B^{\prime} x^{-b}\left(-(1+\mu)+(1+\mu)^{a} a^{-1}+(1+\mu)^{a}(A a)^{-1} r\left(u_{x}\right) u_{x}^{-a}\right)\right\} \\
\leq & \exp \left\{B^{\prime} x^{-b}\left(\left(a^{-1}-1\right)+\frac{1}{2}(a-1) \mu^{2}+O\left(\mu^{3}\right)+(1+\mu)^{a}(A a)^{-1} r\left(u_{x}\right) u_{x}^{-a}\right)\right. \\
& \left.\quad+O\left(\log \mu^{-1}\right)\right\} \\
= & \exp \left\{B x^{-b}\left(1-\frac{1}{2} a \mu^{2}+O\left(\mu^{3}\right)+(1+O(\mu)) A^{-1}(1-a)^{-1} r\left(u_{x}\right) u_{x}^{-a}\right)\right. \\
\quad & \left.\quad O\left(\log \mu^{-1}\right)\right\},
\end{aligned}
$$

where we have used the identity $B^{\prime}\left(a^{-1}-1\right)=B$ which follows from $(2.3)$. Expressing $r\left(u_{x}\right) u_{x}^{-a}$ in terms of $\mu$ using (2.10), the last expression becomes

$$
\exp \left\{B x^{-b}\left(1-\mu^{2}\left(\frac{a}{2}-K^{-2} A^{-1}(1-a)^{-1}\right)+O\left(\mu^{3}\right)+O\left(x^{b} \log \mu^{-1}\right)\right)\right\} .
$$

By (2.8), (2.10) and condition (R2) we have

$$
x^{b} \log \mu^{-1} \ll u_{x}^{-a} \log u_{x}=o\left(u_{x}^{-a} r\left(u_{x}\right)\right)=o\left(\mu^{2}\right) .
$$

Choosing now $K$ sufficiently large in terms of $A$ and $a$ yields

$$
x I_{4} \leq \exp \left\{B x^{-b}\left(1-a \mu^{2} / 4\right)\right\}
$$

for all sufficiently small $x$. By a similar argument we have

$$
x I_{2} \leq \exp \left\{B x^{-b}\left(1-a \mu^{2} / 4\right)\right\}
$$

for all sufficiently small $x$.

We now estimate $I_{3}$. By $(2.1 \mathrm{~b})$ we have

$$
x I_{3}=x \int_{(1-\mu) u_{x}}^{(1+\mu) u_{x}} e^{-x t} P(t) d t \leq x \int_{(1-\mu) u_{x}}^{(1+\mu) u_{x}} e^{-x t+A t^{a}+r(t)} d t
$$

We observe that $t=u_{x}$ maximizes $-x t+A t^{a}$ and, by condition (R2), $r(t) \leq$ $r\left((1+\mu) u_{x}\right)$ for all $t \leq(1+\mu) u_{x}$. Hence, by $(2.7),(2.8)$ and $(2.11)$,

$$
\begin{aligned}
x I_{3} & \leq 2 x \mu u_{x} \exp \left\{-x u_{x}+A u_{x}^{a}+r\left((1+\mu) u_{x}\right)\right\} \\
& \leq 2 x \mu u_{x} \exp \left\{B x^{-b}+(1+\mu)^{a} r\left(B^{\prime} x^{-b-1}\right)\right\}
\end{aligned}
$$

for all sufficiently small $x$. Combining (2.9), (2.12), (2.13) and (2.14), and noting that, by (2.7) and (2.10),

$$
\log \left(x \mu u_{x}\right)=\log \left(B^{\prime} x^{-b} K \sqrt{u_{x}^{-a} r\left(u_{x}\right)}\right)=O\left(\log \left(u_{x}^{a} r\left(u_{x}\right)\right)\right)=O\left(\log x^{-1}\right),
$$

we finally obtain

$$
\begin{aligned}
F(x) \leq & O(x)+O\left(\exp \left\{B x^{-b}-a \mu^{2} / 4\right\}\right) \\
& +\exp \left\{B x^{-b}+(1+\mu)^{a} r\left(B^{\prime} x^{-b-1}\right)+O\left(\log x^{-1}\right)\right\} \\
= & \exp \left\{B x^{-b}+(1+\mu)^{a} r\left(B^{\prime} x^{-b-1}\right)+O\left(\log x^{-1}\right)\right\} \\
\leq & \exp \left\{B x^{-b}+2 r\left(B^{\prime} x^{-b-1}\right)\right\}
\end{aligned}
$$

for all sufficiently small $x$, where in the last step we have used the assumption (R2). This yields the desired estimate $(2.2 \mathrm{~b})$.

Our next result is a Tauberian counterpart to Proposition 1. 
Proposition 2. Let $P(u)$ and $F(x)$ be defined as in Proposition 1. Suppose that for some constants $B, b>0$ and $B^{\prime}=B b$ the inequality

$$
\left|\log F(x)-B x^{-b}\right| \leq r\left(B^{\prime} x^{-b-1}\right)
$$

holds for all sufficiently small $x$, where $r(u)$ is a positive differentiable function satisfying conditions (R1) and (R2) in Proposition 1 with $a=b /(1+b)$. Then

$$
-C \sqrt{u^{a} r(u)} \leq \log P(u)-A u^{a} \leq r(u)
$$

for all sufficiently large $u$, where $A$ and a are determined by (2.3) and $C$ is a constant depending on $B$ and $b$.

Proof. Suppose that (2.15) holds for all sufficiently small $x$. We first prove the upper bound for $\log P(u)$.

Given a positive number $u$, by (2.4) and assumption (2.15) we have

$$
P(u) \leq e^{x u} F(x) \leq \exp \left\{x u+B x^{-b}+r\left(B^{\prime} x^{-b-1}\right)\right\}
$$

for all sufficiently small $x$. We choose

$$
x=x_{u}=\operatorname{Aau}^{-(1-a)},
$$

where $A$ and $a$ are determined by (2.3), and note that with this choice of $x$ we have $u=u_{x}$, where $u_{x}$ is given by (2.6). We assume that $u$ is large enough so that (2.17) holds for $x=x_{u}$. Using (2.7) and (2.8) with $x_{u}$ and $u$ in place of $x$ and $u_{x}$, we see that

$$
x_{u} u+B x_{u}^{-b}=B^{\prime} x_{u}^{-b}\left(1+b^{-1}\right)=A a u_{x}^{a}\left(1+\frac{1-a}{a}\right)=A u_{x}^{a}
$$

and

$$
B^{\prime} x_{u}^{-b-1}=u
$$

Thus, we obtain the upper bound

$$
P(u) \leq \exp \left\{x_{u} u+B x_{u}^{-b}+r\left(B^{\prime} x_{u}^{-b-1}\right)\right\}=\exp \left\{A u^{a}+r(u)\right\}
$$

for all sufficiently large $u$. This implies the upper bound in (2.16). It remains to prove the lower bound.

Let $u_{0}$ be a large positive constant such that (2.21) holds for $u \geq u_{0}$. Assuming that $u \geq 2 u_{0}$, we split the integral defining $F\left(x_{u}\right)$ into four parts as before. Let $I_{1}, I_{2}, I_{3}$ and $I_{4}$ be defined by (2.9) with $x=x_{u}$ and $u_{x}$ replaced by $u$. We let $\mu=\mu(u)=K \sqrt{u^{-a} r(u)}$ be defined as in (2.10), where $K$ is a large positive constant to be chosen later. Using the upper bound (2.21) and arguing exactly as in the proof of Proposition 1, we see that the upper bounds (2.12) and (2.13) for $I_{4}$ and $I_{2}$ remain valid for sufficiently large $u$ with $x_{u}$ and $u$ in place of $x$ and $u_{x}$. We note that, by (2.3), (2.8) and the definition of $\mu$,

$\frac{1}{4} B a \mu^{2} x_{u}^{-b}=\frac{1}{4} B a K^{2} r(u) u^{-a} x_{u}^{-b}=\frac{1}{4} B a K^{2} r(u) \frac{A a}{B^{\prime}}=\frac{1}{4} A a(1-a) K^{2} r\left(B^{\prime} x_{u}^{-b-1}\right)$.

We now choose the constant $K$ large enough so that $A a(1-a) K^{2} / 4 \geq 2$. Then by (2.12) and assumption (2.15), we have

$$
\begin{aligned}
x_{u} I_{4} & \leq \exp \left\{B x_{u}^{-b}-\frac{1}{4} B a \mu^{2} x_{u}^{-b}\right\} \leq \exp \left\{B x_{u}^{-b}-2 r\left(B^{\prime} x_{u}^{-b-1}\right)\right\} \\
& \leq F\left(x_{u}\right) \exp \left\{-r\left(B^{\prime} x_{u}^{-b-1}\right)\right\}=o\left(F\left(x_{u}\right)\right) .
\end{aligned}
$$


Similarly, we see that $x_{u} I_{2}=o\left(F\left(x_{u}\right)\right)$. Hence $x_{u} I_{3} \geq F\left(x_{u}\right)(1+o(1))$. On the other hand, bounding the integral $I_{3}$ trivially, we obtain

$$
x_{u} I_{3} \leq 2 \mu u x_{u} e^{-x_{u}(1-\mu) u} P((1+\mu) u) .
$$

It follows that

$$
P((1+\mu) u) \geq F\left(x_{u}\right) \exp \left\{x_{u}(1-\mu) u-\log \left(2 \mu u x_{u}\right)+o(1)\right\} .
$$

By (2.10), (2.18) and conditions (R1) and (R2) we have

$$
\log \left(2 \mu u x_{u}\right) \ll \log u \ll r(u) .
$$

Thus, using the bound (2.15) for $F\left(x_{u}\right)$, we obtain

$$
P((1+\mu) u) \geq \exp \left\{B x_{u}^{-b}-r\left(B^{\prime} x_{u}^{-b-1}\right)+x_{u}(1-\mu) u+O(r(u))\right\} .
$$

Substituting (2.18), (2.19) and (2.20) into the last expression, we see that

$$
\log P((1+\mu) u) \geq A u^{a}-A a \mu u^{a}+O(r(u))=A(1+\mu)^{a} u^{a}+O\left(\mu u^{a}\right)+O(r(u)) .
$$

The last error term can be omitted since $r(u)=o\left(\sqrt{r(u) u^{a}}\right)=o\left(\mu u^{a}\right)$ by condition (R1) and (2.10). Moreover, by condition (R2), we have $\mu u^{a}=K \sqrt{r(u) u^{a}} \leq$ $K \sqrt{r((1+\mu) u)(1+\mu)^{a} u^{a}}$. It follows that

$$
\log P((1+\mu) u) \geq A(1+\mu)^{a} u^{a}-C \sqrt{r((1+\mu) u)(1+\mu)^{a} u^{a}}
$$

for all sufficiently large $u$. Since $(1+\mu(u)) u$ is a continuous function of $u$ and tends to infinity when $u \rightarrow \infty$, for all sufficiently large $v$, there exists a $u$ such that $v=(1+\mu(u)) u$. Hence we have

$$
\log P(v) \geq A v^{a}-C \sqrt{v^{a} r(v)}
$$

for all sufficiently large $v$. This completes the proof of the proposition.

Proposition 2 is not a complete converse to Proposition 1, as the lower bound in (2.16) is weaker than the lower bound for $\log P(u)$ in Proposition 1. Our next result gives, under a stronger hypothesis, a complete converse.

Proposition 3. Let $P(u)$ and $F(x)$ be defined as in Proposition 1. Suppose that for some constants $B, b>0$ and $B^{\prime}=B b$ the inequality

$$
\left|\log F(x)-B x^{-b}\right| \leq r\left(B^{\prime} x^{-b-1}\right)
$$

holds for all sufficiently small $x$, where $r(u)$ is a positive differentiable function satisfying conditions (R1) and (R2) in Proposition 1 with $a=b /(1+b)$. Suppose that, in addition, $r(u)$ satisfies

$$
r(u) \gg u^{a / 2}
$$

as $u \rightarrow \infty$. Suppose further that the function $G(x)=\log F(x)$ satisfies

$$
G^{\prime}(x)=-B^{\prime} x^{-b-1}+O\left(x^{-1} r\left(B^{\prime} x^{-b-1}\right)\right)
$$

and

$$
G^{\prime \prime}(x) \gg x^{-b-2}
$$

as $x \rightarrow 0$. Then

$$
-C r(u) \leq \log P(u)-A u^{a} \leq r(u)
$$


for all sufficiently large $u$, where $A$ and $a$ are determined by (2.3), and $C$ is a positive constant depending on $B, b$ and the constants implicit in (2.22), (2.23) and condition (R3).

Proof. The upper bound in (2.24) follows from Proposition 2. Therefore it remains to prove the lower bound in (2.24). To this end we use a method of Odlyzko 9].

Given a large number $u$, we let $0<\mu_{1}<\mu_{2}<1$ be positive numbers to be chosen later as functions of $u$. Set $u_{0}=u, u_{1}=\left(1-\mu_{1}\right) u, u_{2}=\left(1-\mu_{2}\right) u$, and let $x_{0}, x_{1}$ and $x_{2}$ be defined by (2.18) with $u=u_{0}, u_{1}$ and $u_{2}$ respectively. Thus, we have $x_{1}=\left(1-\mu_{1}\right)^{-(1-a)} x_{0}$ and $x_{2}=\left(1-\mu_{2}\right)^{-(1-a)} x_{0}$. We consider the function

$$
\begin{gathered}
h(t)=\exp \left\{x_{1} u_{1}-x_{1} t\right\}-\exp \left\{x_{0} u_{0}+x_{1} u_{1}-x_{1} u_{0}-x_{0} t\right\} \\
-\exp \left\{x_{2} u_{2}+x_{1} u_{1}-x_{1} u_{2}-x_{2} t\right\} .
\end{gathered}
$$

Since $x_{0}<x_{1}<x_{2}$, we have for $t \geq u_{0}$

$$
x_{1} u_{1}-x_{1} t \leq x_{1} u_{1}-x_{1} t+\left(x_{1}-x_{0}\right)\left(t-u_{0}\right)=x_{0} u_{0}+x_{1} u_{1}-x_{1} u_{0}-x_{0} t,
$$

and for $t \leq u_{2}$

$$
x_{1} u_{1}-x_{1} t \leq x_{1} u_{1}-x_{1} t+\left(x_{2}-x_{1}\right)\left(u_{2}-t\right)=x_{2} u_{2}+x_{1} u_{1}-x_{1} u_{2}-x_{2} t .
$$

Thus $h(t) \leq 0$ for $t \leq u_{2}$ and $t \geq u_{0}$. Now we let

$$
\begin{aligned}
H & =\int_{0}^{\infty} h(t) d P(t) \\
& =e^{x_{1} u_{1}} F\left(x_{1}\right)-e^{x_{0} u_{0}+x_{1} u_{1}-x_{1} u_{0}} F\left(x_{0}\right)-e^{x_{2} u_{2}+x_{1} u_{1}-x_{1} u_{2}} F\left(x_{2}\right) .
\end{aligned}
$$

Then we see that

$$
\begin{aligned}
H & \leq\left(P\left(u_{0}\right)-P\left(u_{2}\right)\right) \times \max _{u_{2} \leq t \leq u_{0}} h(t) \\
& \leq P\left(u_{0}\right) \times \max _{u_{2} \leq t \leq u_{0}} e^{x_{1} u_{1}-x_{1} t}=P\left(u_{0}\right) e^{x_{1}\left(u_{1}-u_{2}\right)} .
\end{aligned}
$$

Hence, we have

$$
\begin{aligned}
P\left(u_{0}\right) \geq\{ & e^{x_{1} u_{1}} F\left(x_{1}\right)-e^{x_{0} u_{0}+x_{1} u_{1}-x_{1} u_{0}} F\left(x_{0}\right) \\
& \left.-e^{x_{2} u_{2}+x_{1} u_{1}-x_{1} u_{2}} F\left(x_{2}\right)\right\} e^{-x_{1}\left(u_{1}-u_{2}\right)} .
\end{aligned}
$$

We now show that, with a suitable choice of $\mu_{1}$ and $\mu_{2}, e^{x_{0} u_{0}+x_{1} u_{1}-x_{1} u_{0}} F\left(x_{0}\right)+$ $e^{x_{2} u_{2}+x_{1} u_{1}-x_{1} u_{2}} F\left(x_{2}\right) \leq e^{x_{1} u_{1}} F\left(x_{1}\right) / 2$, and thus the last expression has the same order of magnitude as $F\left(x_{1}\right) e^{x_{1} u_{2}}$.

By assumptions (2.22) and (2.23), there are positive constants $C_{1}$ and $C_{2}$ such that

$$
-B^{\prime} x^{-b-1}-C_{1} x^{-1} r\left(B^{\prime} x^{-b-1}\right) \leq G^{\prime}(x) \leq-B^{\prime} x^{-b-1}+C_{1} x^{-1} r\left(B^{\prime} x^{-b-1}\right)
$$

and

$$
G^{\prime \prime}(x) \geq C_{2} x^{-b-2}
$$

when $x$ is sufficiently small. Thus, Taylor's formula yields that

$$
\begin{gathered}
G\left(x_{1}\right)-G\left(x_{0}\right) \geq G^{\prime}\left(x_{0}\right)\left(x_{1}-x_{0}\right)+\frac{1}{2} \min _{x_{0} \leq \xi \leq x_{1}} G^{\prime \prime}(\xi)\left(x_{1}-x_{0}\right)^{2} \\
\geq\left(x_{1}-x_{0}\right)\left(-B^{\prime} x_{0}^{-b-1}-C_{1} x_{0}^{-1} r\left(B^{\prime} x_{0}^{-b-1}\right)\right) \\
+\frac{1}{2} C_{2} x_{1}^{-b-2}\left(x_{1}-x_{0}\right)^{2}
\end{gathered}
$$


We now choose $\mu_{1}$ to be of the form $\mu_{1}=K x_{0}^{b} r\left(B^{\prime} x_{0}^{-b-1}\right)$, where $K$ is a large constant independent of $u_{0}$. We note that, by (2.8) and (2.20), $\mu_{1}$ is also equal to $K B^{\prime}(A a)^{-1} u_{0}^{-a} r\left(u_{0}\right)$. Thus, by condition (R3), we have

$$
\mu_{1} \geq K C_{3} u_{0}^{-a / 2}
$$

for all sufficiently large $u_{0}$, where $C_{3}$ is a positive constant depending on the constant implicit in condition (R3). Moreover, by condition (R1), this choice of $\mu_{1}$ satisfies $\mu_{1} \rightarrow 0$ as $u_{0} \rightarrow \infty$. It follows that when $u_{0}$ is sufficiently large,

$$
x_{1}-x_{0}=x_{0}\left(\left(1-\mu_{1}\right)^{-(1-a)}-1\right)\left\{\begin{array}{l}
\leq 2(1-a) \mu_{1} x_{0}, \\
\geq(1-a) \mu_{1} x_{0} .
\end{array}\right.
$$

Hence, recalling that, by $(2.20), B^{\prime} x_{0}^{-b-1}=u_{0}$, we have from (2.26)

$$
\begin{aligned}
& G\left(x_{1}\right)-G\left(x_{0}\right) \geq-\left(x_{1}-x_{0}\right) u_{0}-2 C_{1}(1-a) \mu_{1} r\left(u_{0}\right) \\
&+\frac{1}{2} C_{2} x_{0}^{-b-2}\left(1-\mu_{1}\right)^{(1-a)(b+2)}(1-a)^{2} \mu_{1}^{2} x_{0}^{2} \\
& \geq-\left(x_{1}-x_{0}\right) u_{0}-2 C_{1}(1-a) \mu_{1} r\left(u_{0}\right)+\frac{1}{4} C_{2}(1-a)^{2} \mu_{1}^{2} x_{0}^{-b}
\end{aligned}
$$

for all sufficiently large $u_{0}$. We now choose $K$ sufficiently large in terms of $B, b, C_{1}$ and $C_{2}$ so that the second term on the right-hand side of the last expression is less than one half of the last term uniformly for all sufficiently large $u_{0}$, and so that the last term is at least $2 \log 4$ (which is possible by (2.8) and (2.27)), i.e., so that

$$
2 C_{1}(1-a) \mu_{1} r\left(B^{\prime} x_{0}^{-b-1}\right) \leq \frac{1}{8} C_{2}(1-a)^{2} \mu_{1}^{2} x_{0}^{-b}
$$

and

$$
\frac{1}{8} C_{2}(1-a)^{2} \mu_{1}^{2} x_{0}^{-b} \geq \log 4
$$

for all sufficiently large $u_{0}$. Then it follows that

$$
\begin{aligned}
G\left(x_{1}\right)-G\left(x_{0}\right) & \geq-\left(x_{1}-x_{0}\right) u_{0}-2 C_{1}(1-a) \mu_{1} r\left(B^{\prime} x_{0}^{-b-1}\right)+\frac{1}{4} C_{2}(1-a)^{2} \mu_{1}^{2} x_{0}^{-b} \\
& \geq-\left(x_{1}-x_{0}\right) u_{0}+\frac{1}{8} C_{2}(1-a)^{2} \mu_{1}^{2} x_{0}^{-b} \\
& \geq-\left(x_{1}-x_{0}\right) u_{0}+\log 4 .
\end{aligned}
$$

Hence

$$
e^{x_{1} u_{1}} F\left(x_{1}\right) \geq 4 e^{x_{0} u_{0}+x_{1} u_{1}-x_{1} u_{0}} F\left(x_{0}\right)
$$

for all sufficiently large $u_{0}$. Similarly, if we choose $\mu_{2}=2 \mu_{1}$, then

$$
e^{x_{1} u_{1}} F\left(x_{1}\right) \geq 4 e^{x_{2} u_{2}+x_{1} u_{1}-x_{1} u_{2}} F\left(x_{2}\right)
$$

for all sufficiently large $u_{0}$. Therefore, by (2.25), (2.28) and (2.29), we have for all sufficiently large $u_{0}$

$$
P\left(u_{0}\right) \geq \frac{1}{2} e^{x_{1} u_{1}} F\left(x_{1}\right) e^{-\left(u_{1}-u_{2}\right) x_{1}}=\frac{1}{2} F\left(x_{1}\right) e^{x_{1} u_{2}}=\exp \left\{G\left(x_{1}\right)+x_{1} u_{2}+O(1)\right\} .
$$


On the other hand, by (2.15) and the definition of $x_{1}$ and $u_{2}$, we have

$$
\begin{aligned}
G\left(x_{1}\right)+x_{1} u_{2} & \geq B x_{1}^{-b}-r\left(B^{\prime} x_{1}^{-b-1}\right)+x_{1} u_{2} \\
& =B\left(1-\mu_{1}\right)^{(1-a) b} x_{0}^{-b}-r\left(B^{\prime} x_{1}^{-b-1}\right)+\left(1-\mu_{1}\right)^{-(1-a)} x_{0}\left(1-2 \mu_{1}\right) u_{0} \\
& =B x_{0}^{-b}+x_{0} u_{0}+O\left(\mu_{1} x_{0}^{-b}\right)-r\left(B^{\prime} x_{1}^{-b-1}\right) \\
& =B x_{0}^{-b}+x_{0} u_{0}+O\left(r\left(B^{\prime} x_{0}^{-b-1}\right)\right)-r\left(B^{\prime} x_{1}^{-b-1}\right)
\end{aligned}
$$

for all sufficiently large $u_{0}$. By condition (R2), we have $r\left(B^{\prime} x_{1}^{-b-1}\right) \leq r\left(B^{\prime} x_{0}^{-b-1}\right)$. It follows that, by (2.19) and (2.20),

$\log P\left(u_{0}\right) \geq G\left(x_{1}\right)+x_{1} u_{2}+O(1) \geq B x_{0}^{-b}+x_{0} u_{0}-C r\left(B^{\prime} x_{0}^{-b-1}\right)=A u_{0}^{a}-C r\left(u_{0}\right)$ for all sufficiently large $u_{0}$, where $C$ is a constant depending on $C_{1}, C_{2}, B$ and $b$. This completes the proof of the proposition.

\section{Some Lemmas on Dirichlet Series and Mellin Transforms}

Let $w(n)$ be a non-negative function defined on the set of positive integers. Let

$$
f_{w}(s)=\sum_{n=1}^{\infty} \frac{w(n)}{n^{s}}
$$

be the Dirichlet series generated by $w(n)$, and suppose that $f_{w}(s)$ has finite abscissa of absolute convergence $\sigma_{a}$. We define $F_{w}(x)$ for $x>0$ by

$$
F_{w}(x)=\prod_{n=1}^{\infty}\left(1-e^{-n x}\right)^{-w(n)} .
$$

We note that in the case $w(n)=\Lambda(n)$, we have $f_{w}(s)=-\zeta^{\prime}(s) / \zeta(s)$, and $F_{w}(x)$ is the generating function for $p_{\Lambda}(n)$.

In this section we will prove some lemmas relating analytic properties of $f_{w}(s)$ to the behavior of $F_{w}(x)$.

Lemma 1. For any $\kappa>\max \left(0, \sigma_{a}\right)$ we have

$$
\log F_{w}(x)=\frac{1}{2 \pi i} \int_{\kappa-i \infty}^{\kappa+i \infty} \Gamma(s) \zeta(1+s) f_{w}(s) x^{-s} d s .
$$

Furthermore, for any $T>1$ we have

$$
\begin{aligned}
\log F_{w}(x)=\frac{1}{2 \pi i} \int_{\kappa-i T}^{\kappa+i T} & \Gamma(s) \zeta(1+s) f_{w}(s) x^{-s} d s \\
& +O\left(\zeta(1+\kappa) f_{w}(\kappa) x^{-\kappa} T^{\kappa+1 / 2} e^{-\pi T / 2}\right),
\end{aligned}
$$

where the $O$-constant is absolute.

Proof. The proof of (3.2) can be found in Meinardus [7, p. 390].

To prove (3.3), we observe that, by Stirling's formula,

$$
\begin{gathered}
\frac{1}{2 \pi i}\left\{\int_{\kappa+i T}^{\kappa+i \infty}+\int_{\kappa-i \infty}^{\kappa-i T}\right\} \Gamma(s) \zeta(1+s) f_{w}(s) x^{-s} d s \\
\ll \zeta(1+\kappa) f_{w}(\kappa) x^{-\kappa} \int_{T}^{\infty} t^{\kappa+1 / 2} e^{-\pi t / 2} d t \\
\ll \zeta(1+\kappa) f_{w}(\kappa) x^{-\kappa} T^{\kappa+1 / 2} e^{-\pi T / 2} .
\end{gathered}
$$

Thus (3.3) follows. 
Lemma 2. For $0<x<1$ and $\operatorname{Re} s>\max \left(0, \sigma_{a}\right)$ we have

$$
\int_{0}^{1} x^{s-1} \log F_{w}(x) d x=\Gamma(s) \zeta(1+s) f_{w}(s)-h_{w}(s),
$$

where $h_{w}(s)$ is an entire function.

Proof. Suppose that $s$ satisfies $\operatorname{Re} s>\max \left(0, \sigma_{a}\right)$. Then

$$
\begin{aligned}
\int_{0}^{1} x^{s-1} \log F_{w}(x) d x & =\left\{\int_{0}^{\infty}-\int_{1}^{\infty}\right\} x^{s-1} \log F_{w}(x) d x \\
& =\left\{\int_{0}^{\infty}-\int_{1}^{\infty}\right\} x^{s-1} \sum_{k=1}^{\infty} \sum_{n=1}^{\infty} \frac{w(n)}{k} e^{-k n x} d x \\
& =\sum_{k=1}^{\infty} \sum_{n=1}^{\infty}\left\{\frac{\Gamma(s) w(n)}{n^{s} k^{s+1}}-\frac{w(n)}{k} \int_{1}^{\infty} x^{s-1} e^{-k n x} d x\right\} \\
& =\Gamma(s) \zeta(1+s) f_{w}(s)-h_{w}(s),
\end{aligned}
$$

where interchanging the order of integration and summation is justified by the absolute convergence of the double series involved. The function $h_{w}(s)$ here is given by

$$
h_{w}(s)=\sum_{k=1}^{\infty} \sum_{n=1}^{\infty} \frac{w(n)}{k} \int_{1}^{\infty} x^{s-1} e^{-k n x} d x=\sum_{k=1}^{\infty} \sum_{n=1}^{\infty} \frac{w(n)}{k^{1+s} n^{s}} \Gamma(s, k n),
$$

where $\Gamma(s, u)=\int_{u}^{\infty} x^{s-1} e^{-x} d x$ is the incomplete Gamma function. Using the estimate

$$
|\Gamma(s, u)| \ll_{\sigma_{1}, \sigma_{2}} \int_{u}^{\infty} e^{-x / 2} d x \ll_{\sigma_{1}, \sigma_{2}} e^{-u / 2} \quad\left(\sigma_{1} \leq \operatorname{Re} s \leq \sigma_{2}, u \geq 1\right)
$$

and the bound $w(n) \ll n^{\sigma_{a}+1}$ (which follows from the convergence of $f_{w}(s)$ at $\left.s=\sigma_{a}+1\right)$, we see that the double series defining $h_{w}(s)$ converges uniformly in any strip $\sigma_{1} \leq \operatorname{Re} s \leq \sigma_{2}$, and hence represents an entire function of $s$.

Lemma 3 (Landau). (i) Let $g(n)$ be a function defined on the set of positive integers and of constant sign for all sufficiently large $n$. Suppose that the Dirichlet series

$$
\sum_{n=1}^{\infty} g(n) n^{-s}
$$

has finite abscissa of absolute convergence $\sigma_{a}$. Then $s=\sigma_{a}$ is a singularity of the function represented by the Dirichlet series.

(ii) Let $g(x)$ be an integrable function on $[0,1]$ and of constant sign for all suffciently small $x$. Suppose that the Dirichlet integral

$$
\int_{0}^{1} g(x) x^{s-1} d x
$$

has finite abscissa of absolute convergence $\sigma_{a}$. Then $s=\sigma_{a}$ is a singularity of the function represented by the Dirichlet integral. 
Proof. Part (i) is the classical version of a well-known theorem of Landau (see, e.g., Ingham [5, p. 88]). To obtain part (ii), we note that the given integral can be written as

$$
\int_{1}^{\infty} h(u) u^{-s} d u
$$

with $h(u)=g(1 / u) / u$ and $x=1 / u$. By the integral version of Landau's lemma (Ingham [5 p. 88]) the last integral has a singularity at $s=\sigma_{a}$.

Lemma 4. Assume that for some constants $B>0$ and $b>0$

$$
\log F_{w}(x)=B x^{-b}\{1+o(1)\}
$$

as $x \rightarrow 0+$. Then the Dirichlet series $f_{w}(s)$ has abscissa of convergence $b$ and satisfies

$$
f_{w}(s)=\frac{1}{s-b}\left\{\frac{B}{\Gamma(b) \zeta(1+b)}+o\left(\frac{|s-b|}{\sigma-b}\right)\right\}
$$

as $s \rightarrow b$, while $\sigma=\operatorname{Re} s>b$.

Proof. By Lemma 2 we have

$$
f_{w}(s)=\frac{1}{\Gamma(s) \zeta(1+s)}\left\{\int_{0}^{1} x^{s-1} \log F_{w}(x) d x+h_{w}(s)\right\},
$$

where $h_{w}(s)$ is an entire function. On the other hand, the hypothesis (3.4) implies that

$$
\begin{aligned}
\int_{0}^{1} x^{s-1} \log F_{w}(x) d x & =B \int_{0}^{1} x^{s-b-1} d x+o\left(\int_{0}^{1} x^{\sigma-b-1} d x\right) \\
& =\frac{B}{s-b}+o\left(\frac{1}{\sigma-b}\right)
\end{aligned}
$$

as $\sigma \rightarrow b+$. Combining these two estimates yields (3.5).

To show that the Dirichlet series $f_{w}(s)$ has abscissa of absolute convergence $b$, we observe that, by (3.6) and (3.7), $f_{w}(s)$ is analytic on the half-plane $\{s: \operatorname{Re} s>b\}$, but has a singularity at the real point $s=b$. Since $w(n)$ is non-negative, Lemma 3 implies that $f_{w}(s)$ has abscissa of absolute convergence $b$.

Lemma 5. Suppose that

$$
g_{w}(s)=f_{w}(s)-\frac{B}{\Gamma(b) \zeta(1+b)} \frac{1}{s-b}
$$

can be meromorphically continued to a half-plane $\left\{s: \operatorname{Re} s>\sigma_{0}\right\}$, where $\sigma_{0}<b$. Let $\theta$ be the least upper bound of the real parts of the singularities of $g_{w}(s)$, and suppose that $g_{w}(s)$ is analytic at the real point $s=\theta$. Then, for any $\epsilon>0$,

$$
\log F_{w}(x)-B x^{-b}=\Omega_{ \pm}\left(x^{-(\theta-\epsilon)}\right) \quad \text { as } x \rightarrow 0 .
$$

Proof. Let $\epsilon$ be given and consider the function

$$
\Phi(x)=\log F_{w}(x)-B x^{-b}-x^{-(\theta-\epsilon)}
$$

with Mellin transform

$$
\phi(s)=\int_{0}^{1} x^{s-1} \Phi(x) d x .
$$


Let $\sigma_{a}$ be the abscissa of absolute convergence of $\phi(s)$. By Lemma 2, we have for $\operatorname{Re} s>b$

$$
\phi(s)=\Gamma(s) \zeta(1+s) f_{w}(s)-h_{w}(s)-\frac{B}{s-b}-\frac{1}{s-(\theta-\epsilon)},
$$

where $h_{w}(s)$ is an entire function. By the definition of $\theta$, for any $\delta>0$, the righthand side of (3.8) has singularities on the half-plane $\{s: \operatorname{Re} s>\theta-\delta\}$; thus we have

$$
\sigma_{a} \geq \theta
$$

Now, if $\Phi(x) \leq 0$ for all sufficiently small $x$, then Lemma 3 implies that the real point $s=\sigma_{a}$ is a singularity of $\phi(s)$. But by the assumption of Lemma 5 , $\phi(s)$ is analytic at the real point $s=\theta$ and has no singularities to the right of $s=\theta$. Thus $\sigma_{a}<\theta$, which contradicts (3.9). It follows that $\Phi(x)$ changes sign infinitely often, i.e., $\log F_{w}(x)-B x^{-b}=\Omega_{+}\left(x^{-(\theta-\epsilon)}\right)$. A similar argument gives $\log F_{w}(x)-B x^{-b}=\Omega_{-}\left(x^{-(\theta-\epsilon)}\right)$.

\section{Proof of Theorems 1-3}

We begin by proving two lemmas which show that the function $p_{\Lambda}(n)$ in our theorems can be replaced by its summatory function $P_{\Lambda}(u)=\sum_{n \leq u} p_{\Lambda}(n)$.

Lemma 6. For any integer $k \geq 15$ and $n \geq 0$, we have $p_{\Lambda}(n+k) \geq p_{\Lambda}(n)$.

Proof. We first observe that, for any odd prime power $q$,

$$
\begin{aligned}
& \sum_{n=0}^{\infty}\left(p_{\Lambda}(n+q)-p_{\Lambda}(n)\right) x^{n}+\sum_{j=0}^{q-1} p_{\Lambda}(j) x^{-q+j} \\
& \quad=\left(x^{-q}-1\right) \prod_{m=1}^{\infty}\left(1-x^{m}\right)^{-\Lambda(m)}=x^{-q}\left(1-x^{q}\right)^{-(\Lambda(q)-1)} \prod_{m \neq q}\left(1-x^{m}\right)^{-\Lambda(m)}
\end{aligned}
$$

Since $\Lambda(q)-1>0$ when $q$ is an odd prime power, expanding each factor in the last expression yields a series with non-negative coefficients. Thus we have $p_{\Lambda}(n+q) \geq$ $p_{\Lambda}(n)$ for all $n \geq 0$. Iterating this inequality with $q=3$ and $q=5$, we see that $p_{\Lambda}(n+3 k+5 l) \geq p_{\Lambda}(n)$ for all non-negative integers $n, k$ and $l$. Since every integer $\geq 15$ can be written as a linear combination of 3 and 5 with non-negative coefficients, the lemma follows.

Lemma 7. Let $n \in \mathbf{N}$. Let $r(u)$ be a function satisfying $r(u)(\log u)^{-1} \rightarrow \infty$ as $u \rightarrow \infty$, and $r(u+v) \ll r(u)$ uniformly for all sufficiently large $u$ and $0 \leq v \leq 15$. Then

$$
\log p_{\Lambda}(n)=2 \sqrt{\zeta(2)} n^{1 / 2}+O(r(n))
$$

as $n \rightarrow \infty$ if and only if

$$
\log P_{\Lambda}(u)=2 \sqrt{\zeta(2)} u^{1 / 2}+O(r(u))
$$

as $u \rightarrow \infty$. A similar statement holds if $O$ is replaced by $\Omega_{ \pm}$.

Proof. By Lemma 6, we have

$$
p_{\Lambda}(n) \leq P_{\Lambda}(n)=\sum_{k=0}^{n} p_{\Lambda}(k) \leq(n+1) p_{\Lambda}(n+15)
$$


for all integers $n \geq 0$. Suppose that, for some positive constant $C$,

$$
2 \sqrt{\zeta(2)} u^{1 / 2}-C r(u) \leq \log P_{\Lambda}(u) \leq 2 \sqrt{\zeta(2)} u^{1 / 2}+C r(u)
$$

for all sufficiently large $u$. Then (4.1) implies that

$2 \sqrt{\zeta(2)}(n-15)^{1 / 2}-\log (n-14)-C r(n-15) \leq \log p_{\Lambda}(n) \leq 2 \sqrt{\zeta(2)} n^{1 / 2}+\operatorname{Cr}(n)$

for all sufficiently large integers $n$. Hence, by the assumptions on $r(u)$, we obtain

$$
\log p_{\Lambda}(n)=2 \sqrt{\zeta(2)} n^{1 / 2}+O(r(n)) .
$$

The proof of the converse implication and of the analogous implications between $\Omega_{ \pm}$-estimates is similar.

By Lemma 7, it suffices to prove Theorems 1-3 with $P_{\Lambda}(u)$ in place of $p_{\Lambda}(n)$.

Proof of Theorem 1. By the Vinogradov-Korobov zero-free region for the Riemann zeta function (Titchmarsh [15] p. 135]), we have $\zeta(s) \neq 0$ for $s=\sigma+i t$ satisfying

$$
\sigma \geq \eta(t)=1-\frac{C}{\left(\log t^{*}\right)^{2 / 3}\left(\log _{2} t^{*}\right)^{1 / 3}},
$$

where $\log _{k}$ is the $k$ times iterated logarithm, $t^{*}=\max (10,|t|)$, and $C$ is a positive constant. Furthermore, in the same region we have

$$
\frac{\zeta^{\prime}(s)}{\zeta(s)} \ll \log t^{*},
$$

provided $|s-1| \geq \delta$ for some positive constant $\delta$.

Applying Lemma 1 with $w(n)=\Lambda(n)$ and noting that in this case $f_{w}(s)=$ $-\zeta^{\prime}(s) / \zeta(s)$, we obtain, for $0<x<1$,

$$
\log F_{\Lambda}(x)=-\frac{1}{2 \pi i} \int_{\kappa-i T}^{\kappa+i T} \Gamma(s) \zeta(1+s) \frac{\zeta^{\prime}(s)}{\zeta(s)} x^{-s} d s+O\left(e^{-T} \frac{\zeta^{\prime}(\kappa)}{\zeta(\kappa)} x^{-\kappa}\right),
$$

where $1<\kappa<2$ and $T>10$ are positive numbers to be chosen later. We shift the path of integration to the path consisting of the segments $\gamma_{1}:\{\sigma-i T: \kappa \geq$ $\sigma>\eta(T)\}, \gamma_{2}:\{\eta(t)+i t:-T \leq t<-10\}, \gamma_{3}:\{\eta(t)+i t:-10 \leq t<10\}$, $\gamma_{4}:\{\eta(t)+i t: 10 \leq t<T\}$ and $\gamma_{5}:\{\sigma+i T: \eta(t) \leq \sigma \leq \kappa\}$.

The estimate

$$
|\Gamma(s)| \ll(1+|t|)^{\sigma+1 / 2} e^{-\pi|t| / 2} \ll e^{-|t|} \quad\left(\frac{1}{2} \leq \sigma \leq 2, t \in \mathbf{R}\right)
$$

(which follows from Stirling's formula) and (4.2) imply that

$$
\int_{\gamma_{1,5}} \ll e^{-T}(\log T) x^{-\kappa}
$$

and

$$
\int_{\gamma_{2,4}} \ll \int_{10}^{T} e^{-t}(\log t) x^{-\eta(t)} d t \ll x^{-\eta(T)}
$$

We also have

$$
\int_{\gamma_{3}} \ll x^{-\eta(10)}
$$


Thus, we obtain

$$
\log F_{\Lambda}(x)=\zeta(2) x^{-1}+O\left(e^{-T} \frac{\zeta^{\prime}(\kappa)}{\zeta(\kappa)} x^{-\kappa}\right)+O\left(e^{-T}(\log T) x^{-\kappa}\right)+O\left(x^{-\eta(T)}\right) .
$$

We now assume that $x$ is sufficiently small so that $\log _{3} x^{-1}$ is defined and $\geq 1$. We set $\kappa=1+1 / \log x^{-1}$ and

$$
T=\frac{C \log x^{-1}}{\left(\log _{2} x^{-1}\right)^{2 / 3}\left(\log _{3} x^{-1}\right)^{1 / 3}},
$$

where $C$ is the constant appearing in the definition of $\eta(t)$. Then $x^{-\kappa}=e x^{-1}$ and $\zeta^{\prime}(\kappa) / \zeta(\kappa) \ll \log x^{-1}$. Hence, noting that

$$
1-\eta(T) \sim \frac{C}{\left(\log _{2} x^{-1}\right)^{2 / 3}\left(\log _{3} x^{-1}\right)^{1 / 3}}
$$

as $x \rightarrow 0$, we obtain from $(4.3)$

$$
\log F_{\Lambda}(x)=\zeta(2) x^{-1}+O\left(x^{-1} \exp \left\{-\frac{(C / 2) \log x^{-1}}{\left(\log _{2} x^{-1}\right)^{2 / 3}\left(\log _{3} x^{-1}\right)^{1 / 3}}\right\}\right) .
$$

We now apply Proposition 2 with $B=B^{\prime}=\zeta(2), b=1$ and the quantities $A$ and $a$ determined by (2.3), i.e.,

$$
a=1 / 2 \quad \text { and } \quad A=a^{-1}(B a /(1-a))^{1-a}=2 \sqrt{\zeta(2)}
$$

Setting $y=B^{\prime} x^{-b-1}=\zeta(2) x^{-2}$ and

$$
r(y)=y^{1 / 2} \exp \left\{-\frac{(C / 5) \log y}{\left(\log _{2} y\right)^{2 / 3}\left(\log _{3} y\right)^{1 / 3}}\right\},
$$

it easily follows from (4.4) that

$$
\left|\log F_{\Lambda}(x)-\zeta(2) x^{-1}\right| \leq r\left(\zeta(2) x^{-2}\right)
$$

for all sufficiently small $x$. Hence, by Proposition 2, we may conclude that, with $c=C / 10$,

$$
\begin{aligned}
\log P_{\Lambda}(u) & =A u^{a}+O\left(\sqrt{r(u) u^{a}}\right) \\
& =2 \sqrt{\zeta(2)} u^{1 / 2}+O\left(u^{1 / 2} \exp \left\{-\frac{c \log u}{\left(\log _{2} u\right)^{2 / 3}\left(\log _{3} u\right)^{1 / 3}}\right\}\right) .
\end{aligned}
$$

This completes the proof of Theorem 1.

Proof of Theorem 2. We will prove only the $\Omega_{-}$-estimate; the $\Omega_{+}$-estimate can be proved similarly.

Applying the corollary to Proposition 1 with $P(u)=P_{\Lambda}(u), F(x)=F_{\Lambda}(x)$, $A=2 \sqrt{\zeta(2)}, a=1 / 2, B=B^{\prime}=\zeta(2), b=1$ and $r(u)=u^{1 / 4}$, we see that it suffices to show that

$$
\log F_{\Lambda}(x)-\zeta(2) x^{-1}=\Omega_{-}\left(x^{-1 / 2}\right) .
$$

Let $\theta$ be the least upper bound of the real parts of the zeros of the Riemann zeta function. If the Riemann Hypothesis is false, i.e., if $\theta>1 / 2$, we apply Lemma 5 with $w(n)=\Lambda(n), f_{w}(s)=-\zeta^{\prime}(s) / \zeta(s), B=\zeta(2)$ and $b=1$. The function $g_{w}(s)$ in Lemma 5 then is given by $-\zeta^{\prime}(s) / \zeta(s)-1 /(s-1)$. Thus $g_{w}(s)$ is a meromorphic function in $\operatorname{Re} s>0$, whose singularities are exactly the zeros of $\zeta(s)$. In particular, the least upper bound of the real parts of these singularities is equal to the least 
upper bound $\theta$ of the real parts of the zeros of $\zeta(s)$. Moreover, since $\zeta(s)$ has no zeros on the real line, $g_{w}(s)$ is analytic at $s=\theta$. Thus, Lemma 5 implies that, for any $\epsilon>0$,

$$
\log F_{\Lambda}(x)-\zeta(2) x^{-1}=\Omega_{ \pm}\left(x^{-(\theta-\epsilon)}\right) .
$$

In particular, we can choose $\epsilon=\theta-1 / 2$ and the assertion of Theorem 2 follows. We therefore assume that the Riemann Hypothesis is true.

Let $1 / 2+i \gamma$ be one of the non-trivial zeros of the Riemann zeta function and let $m$ be its multiplicity. Let $C$ be a positive constant such that

$$
C<m|\Gamma(1 / 2+i \gamma) \zeta(3 / 2+i \gamma)|
$$

and define

$$
\Phi(x)=\log F_{\Lambda}(x)-\zeta(2) x^{-1}+C x^{-1 / 2} .
$$

If (4.6) is false, then there exists $x_{0}>0$ such that $\Phi(x) \geq 0$ holds for all $x \leq x_{0}$. By Lemma 3, the integral

$$
\phi(s)=\int_{0}^{1} \Phi(x) x^{s-1} d x
$$

has a singularity at the real point $s=\sigma_{a}$, the abscissa of absolute convergence of $\phi(s)$. On the other hand, applying Lemma 2 with $f_{w}(s)=-\zeta^{\prime}(s) / \zeta(s)$, we obtain

$$
\phi(s)=-\Gamma(s) \zeta(1+s) \frac{\zeta^{\prime}(s)}{\zeta(s)}-h_{\Lambda}(s)-\frac{\zeta(2)}{s-1}+\frac{C}{s-1 / 2},
$$

where $h_{\Lambda}(s)$ is an entire function. Since the last expression has no singularity on the real axis to the right of $1 / 2$, the abscissa of absolute convergence $\sigma_{a}$ of the integral $\phi(s)$ must be $\leq 1 / 2$. For $\sigma>1 / 2$ we have

$$
\begin{aligned}
|\phi(\sigma+i \gamma)| & \leq \int_{x_{0}}^{1}|\Phi(x)| x^{\sigma-1} d x+\int_{0}^{x_{0}} \Phi(x) x^{\sigma-1} d x \\
& =\int_{x_{0}}^{1}\{|\Phi(x)|-\Phi(x)\} x^{\sigma-1} d x+\int_{0}^{1} \Phi(x) x^{\sigma-1} d x \\
& \leq \int_{x_{0}}^{1} 2|\Phi(x)| x^{-1 / 2} d x+\phi(\sigma) .
\end{aligned}
$$

We multiply both sides by $\sigma-1 / 2$ and let $\sigma \rightarrow 1 / 2+$. The right-hand side becomes $\lim _{\sigma \rightarrow 1 / 2+}(\sigma-1 / 2) \phi(\sigma)$, which, by (4.8), is equal to

$$
\lim _{\sigma \rightarrow 1 / 2+}(\sigma-1 / 2)\left|-\Gamma(\sigma) \zeta(1+\sigma) \frac{\zeta^{\prime}(\sigma)}{\zeta(\sigma)}-\frac{\zeta(2)}{\sigma-1}+\frac{C}{\sigma-1 / 2}\right|=C .
$$

On the other hand, applying (4.8) to $\phi(\sigma+i \gamma)$, we see that the left-hand side of (4.9) becomes (after multiplying by $\sigma-1 / 2$ and letting $\sigma \rightarrow 1 / 2+$ )

$$
\begin{aligned}
\lim _{\sigma \rightarrow 1 / 2+}(\sigma-1 / 2) \mid & -\Gamma(\sigma+i \gamma) \zeta(1+\sigma+i \gamma) \frac{\zeta^{\prime}(\sigma+i \gamma)}{\zeta(\sigma+i \gamma)} \\
& -\frac{\zeta(2)}{\sigma-1+i \gamma}+\frac{C}{\sigma-1 / 2+i \gamma}|=m| \Gamma(1 / 2+i \gamma) \zeta(3 / 2+i \gamma) \mid .
\end{aligned}
$$

Hence $m|\Gamma(1 / 2+i \gamma) \zeta(3 / 2+i \gamma)| \leq C$, which contradicts (4.7). Therefore (4.6) holds, and the proof of Theorem 2 is complete. 
Proof of Theorem 3. Let $\theta$ be the least upper bound for the real parts of the zeros of the Riemann zeta function. It suffices to show that if $\alpha$ is a real number such that the estimate

$$
\log P_{\Lambda}(u)=2 \sqrt{\zeta(2)} u^{1 / 2}+O\left(u^{\alpha / 2}\right)
$$

holds as $u \rightarrow \infty$, then $\alpha \geq \theta$.

By Proposition 1, (4.10) implies that

$$
\log F_{\Lambda}(x)=\zeta(2) x^{-1}+O\left(x^{-\alpha}\right) .
$$

Thus the integral

$$
\phi(s)=\int_{0}^{1} x^{s-1}\left\{\log F_{\Lambda}(x)-\zeta(2) x^{-1}\right\} d x
$$

defines an analytic function on the half-plane $\{s: \operatorname{Re} s>\alpha\}$. On the other hand, by Lemma 2 we have, for $\operatorname{Re} s>1$,

$$
\phi(s)=-\Gamma(s) \zeta(1+s) \frac{\zeta^{\prime}(s)}{\zeta(s)}-\frac{\zeta(2)}{s-1}-h_{\Lambda}(s),
$$

where $h_{\Lambda}(s)$ is an entire function. Since $\phi(s)$ is analytic in Res $>\alpha$, the latter relation remains valid in this larger region, and the right-hand side of (4.11) is analytic in $\operatorname{Res}>\alpha$. Therefore $\zeta(s)$ cannot have zeros on the half-plane $\{s: \operatorname{Re} s>$ $\alpha$, i.e., we have $\theta \leq \alpha$, as claimed.

\section{A New Proof of Theorem B}

Let $\theta$ be the least upper bound for the real parts of the zeros of the Riemann zeta function. We may assume $1 / 2 \leq \theta<1$, for if $\theta=1$, the asserted estimate follows from Theorem 1.

By Lemma 1 we have, for $0<x<1$ and $\kappa>1$,

$$
\log F_{\Lambda}(x)=-\frac{1}{2 \pi i} \int_{\kappa-i \infty}^{\kappa+i \infty} \Gamma(s) \zeta(1+s) \frac{\zeta^{\prime}(s)}{\zeta(s)} x^{-s} d s .
$$

By standard arguments (as, for example, in the proof of Theorems 28 of Ingham [5]) we now shift the line of integration to the vertical line $\operatorname{Re} s=-1 / 2$, taking into account the residues of the integrand at $s=1,0$ and at the zeta-zeros. This gives

$$
\begin{aligned}
\log F_{\Lambda}(x)=\zeta(2) x^{-1} & -\sum_{\rho} \Gamma(\rho) \zeta(1+\rho) x^{-\rho}-\frac{\zeta^{\prime}(0)}{\zeta(0)} \log x^{-1} \\
& -\frac{1}{2 \pi i} \int_{-1 / 2-i \infty}^{-1 / 2+i \infty} \Gamma(s) \zeta(1+s) \frac{\zeta^{\prime}(s)}{\zeta(s)} x^{-s} d s
\end{aligned}
$$

where $\rho$ runs over non-trivial zeta-zeros, counted with multiplicities.

By the symmetry of the zeta-zeros and the assumption that $\theta<1$, we have

$$
|\zeta(1+\rho)| \leq \zeta(1+(1-\theta)) \ll 1
$$

for all zeta-zeros $\rho$. Using Stirling's formula and the bound

$$
\sum_{T \leq \operatorname{Im} \rho \leq T+1} 1 \ll \log T,
$$


we obtain

$$
\sum_{\rho} \Gamma(\rho) \zeta(1+\rho) x^{-\rho} \ll \sum_{\rho=\beta+i \gamma} e^{-|\gamma|} x^{-\beta} \ll x^{-\theta} .
$$

Also, for $\operatorname{Re} s=-1 / 2$ we have $\zeta^{\prime}(s) / \zeta(s) \ll \log (|s|+2)$ and $\zeta(1+s) \ll(|s|+1)^{1 / 2}$ (Theorems 9 and 27 in [5]). Thus

$$
\begin{aligned}
\int_{-1 / 2-i \infty}^{-1 / 2+i \infty} & \Gamma(s) \zeta(1+s) \frac{\zeta^{\prime}(s)}{\zeta(s)} x^{-s} d s \\
& \ll x^{1 / 2} \int_{-\infty}^{\infty} e^{-|t|}(|t|+1)^{1 / 2} \log (|t|+2) d t \ll x^{1 / 2} .
\end{aligned}
$$

Hence, we obtain

$$
\log F_{\Lambda}(x)=\zeta(2) x^{-1}+O\left(x^{-\theta}\right) .
$$

Similarly, we have, for $\kappa>1$,

$$
\begin{aligned}
\frac{d}{d x} \log F_{\Lambda}(x) & =\frac{1}{2 \pi i} \int_{\kappa-i \infty}^{\kappa+i \infty} s \Gamma(s) \zeta(1+s) \frac{\zeta^{\prime}(s)}{\zeta(s)} x^{-s-1} d s \\
& =-\zeta(2) x^{-2}+O\left(x^{-(\theta+1)}\right)
\end{aligned}
$$

and

$$
\begin{aligned}
\frac{d^{2}}{d x^{2}} \log F_{\Lambda}(x) & =-\frac{1}{2 \pi i} \int_{\kappa-i \infty}^{\kappa+i \infty} s(1+s) \Gamma(s) \zeta(1+s) \frac{\zeta^{\prime}(s)}{\zeta(s)} x^{-s-2} d s \\
& =2 \zeta(2) x^{-3}+O\left(x^{-(\theta+2)}\right) .
\end{aligned}
$$

By (5.1)-(5.3), the function $F(x)=F_{\Lambda}(x)$ satisfies the hypotheses (2.15), (2.22) and (2.23) of Proposition 3 with $b=1, B=B^{\prime}=\zeta(2)$ and $r(u)=K u^{\theta / 2}$, where $K$ is a sufficiently large constant. Moreover, the function $r(u)$ satisfies conditions (R1), (R2) and (R3) of that proposition. Hence the conclusion (2.24) of Proposition 3 holds with $A$ and $a$ given by (4.5). Therefore

$$
\log P_{\Lambda}(u)=A u^{a}+O(r(u))=2 \sqrt{\zeta(2)} u^{1 / 2}+O\left(u^{\theta / 2}\right)
$$

as $u \rightarrow \infty$, which is the claimed result.

\section{ACKNOWLEDGMENT}

The author wishes to thank Professor A. Hildebrand for helpful advice on the exposition of the paper.

\section{REFERENCES}

1. N. A. Brigham, On a certain weighted partition function, Proc. Amer. Math. Soc. 1 (1950), 192-204. MR 11:582C

2. G. A. Freiman, Inverse problems of the additive theory of numbers, Izv. Akad. SSSR. Ser. Mat. 19 (1955), 275-284. (Russian) MR 17:239c

3. G. H. Hardy and S. Ramanujan, Asymptotic formulae in combinatory analysis, Proc. London Math. Soc. (2) 17 (1918), 75-115.

4. A. E. Ingham, A Tauberian theorem for partitions, Ann. of Math. (2) 42 (1941), 1075-1090. MR 3:166a

5. MR 91f: 11064

6. E. E. Kohlbecker, Weak asymptotic properties of partitions, Trans. Amer. Math. Soc $\mathbf{8 8}$ (1958), 346-365. MR 20:2309 
7. G. Meinardus, Asymptotische Aussagen über Partitionen, Math. Z. 59 (1954), 388-398. MR 16:17e

8. — Über Partitionen mit Differenzenbedingungen, Math. Z. 61 (1954), 289-302. MR 16:905a

9. A. M. Odlyzko, Explicit Tauberian estimates for functions with positive coefficients, J. Comput. Appl. Math. 41 (1992), 187-197. MR 94f:11086

10. B. Richmond, Asymptotic relations for partitions, J. Number Theory 7 (1975), 389-405. MR 52:3095

11. _ A general asymptotic result for partitions, Canad. J. Math. 27 (1975), 1083-1091. MR 52:5604

12. K. F. Roth and G. Szekeres, Some asymptotic formulae in the theory of partitions, Quart. J. Math., Oxford Ser. (2) 5 (1954), 241-259. MR 16:797b

13. W. Schwarz, Schwache asymptotische Eigenschaften von Partitionen, J. Reine Angew. Math. 232 (1968), 1-16. MR 38:4433

14. - Asymptotische Formeln für Partitionen, J. Reine Angew. Math. 234 (1969), 174-178. MR 40:7217

15. E. C. Titchmarsh, The theory of the Riemann zeta-function, 2nd ed., The Clarendon Press, Oxford University Press, New York, 1986. MR 88c:11049

Department of Mathematics, University of Illinois, Urbana, Illinois 61801

E-mail address: yfyang@math.uiuc.edu 